\title{
Una aproximación a las tipologías en la investigación sociológica a partir de los estilos de vida de homosexuales masculinos en Lima
}

\author{
An approximation to typologies in sociological research based on \\ the lifestyles of masculine homosexuals in Lima
}

\author{
Mauricio Rentería \\ Instituto de Estudios Peruanos \\ mrenteria@iep.org.pe (PERÚ)
}

Recibido: 25.012016

Aceptado: 25.11 .2016

\section{RESUMEN}

En este artículo presentamos una vía heurística para la construcción de tipologías en la investigación sociológica. Mostramos las principales características de las tipologías, su relación con los conceptos de sentido común y su lugar dentro de los conceptos científicos. A su vez, con los datos de una encuesta realizada a fines del 2014 en el marco de una investigación en curso sobre las formas de subjetivación de la homosexualidad en Lima, presentamos una tipología de los estilos de vida de los hombres que practican el homoerotismo. Con esta exposición buscamos reconstruir el modus operandi de la elaboración de tipologías, en tanto articulación de los niveles teórico y técnico-metodológico de la investigación. Para el primer nivel exponemos un breve marco para el análisis, mientras que para el segundo efectuamos dos técnicas de análisis estadístico: el análisis de correspondencias múltiples (ACM) y la clasificación ascendente jerárquica $(\mathrm{CAJ})$.

\section{PALABRAS CLAVE}

Tipología, construcción del objeto de estudio, tipo extraído, estilos de vida, homosexuales masculinos. 


\begin{abstract}
In this paper we present a heuristic pathway for the construction of typologies in sociological research. We show the main characteristics of the types, their relationship with the concepts of common sense and their place within scientific concepts. At the same time, using data from a survey conducted in late 2014 as part of an ongoing research about the forms of subjectivation of homosexuality in Lima, we present a typology of the lifestyles of men who practice homoeroticism. With this presentation we seek to reconstruct the modus operandi of the development of typologies, as it articulates the theoretical and technical-methodological research levels. For the first level we draw upon a brief framework for the analysis, as for the second we use two techniques of statistical analysis: the multiple correspondence analysis (MCA) and the hierarchical ascendant classification (HAC).
\end{abstract}

\title{
KEY WORDS
}

Typology, construction of the object of study, extracted type, lifestyles, masculine homosexuals.

\section{INTRODUCCIÓN}

Las tipologías han estado presentes en las más diversas teorías y corrientes de la sociología: desde la teoría marxista de las clases sociales y las formas de dominación en Weber; hasta los tipos de acción en la teoría de la acción comunicativa de Habermas y las formas de capital en la teoría de los campos sociales de Bourdieu. Su reiterado uso se explica en parte por su plasticidad como recurso metodológico, que se evidencia en las diferentes funciones que puede cumplir dentro de la investigación empírica. Como sostiene Velasco (2014), las tipologías pueden servir: i) como medio para la selección de casos, ii) como un instrumento auxiliar en la organización de la información, iii) como resultado intermedio en la construcción del objeto de estudio, y iv) como vía en la construcción de conceptos o sistemas teóricos (Velasco 2014).

Sin embargo, su extensa utilización en la investigación sociológica no ha anidado en las contribuciones para su reflexión (López 1996; Velasco 2014). En este artículo no pretendemos brindar un análisis exhaustivo de las tipologías, para lo que remitimos al lector a la bibliografía disponible al respectoํ. Más bien, pretendemos exponer, de manera pormenorizada, una forma específica de construcción de tipologías, empíricamente fundamentada y apoyada en el uso de dos técnicas de análisis estadístico: el análisis de correspondencias

${ }^{1}$ Para desarrollos sobre el recurso de la tipología en la investigación social, remitimos al lector a los trabajos de McKinney (1969), Lozares (1990), López (1996), Velasco (2014) y Cohen y Gómez (2011). 
múltiples (ACM) y la clasificación ascendente jerárquica (CAJ). La exposición se enmarca en una investigación en curso sobre las formas de subjetivación de la homosexualidad masculina en la ciudad de Lima. En particular, desarrollaremos la parte cuantitativa de la investigación, la cual se enfoca en la identificación y análisis de los distintos estilos de vida de quienes componen esta colectividad ${ }^{2}$.

En la primera parte del artículo desarrollamos una breve introducción a la función y los usos de las tipologías en la investigación social. Aquí expondremos sus principales características, su relación con los conceptos de sentido común y su lugar dentro de los conceptos científicos. Nuestro principal referente en esta sección es la "tipología constructiva" de McKinney (1969) y prestamos especial atención a la distinción que establece entre "tipos ideales" y "tipos extraídos" debido a su importancia para el análisis que desarrollaremos en la siguiente sección. En la segunda parte, que constituye el grueso del artículo, presentamos los pasos para la elaboración de un tipo extraído en base a los datos de una encuesta en el marco de una investigación en curso sobre las formas de subjetivación de homosexuales masculinos en la ciudad de Lima. Buscamos reconstruir el modus operandi de la elaboración de tipologías sociológicas, como una articulación de los niveles analíticos y técnico-metodológicos de la investigación. Finalmente se exponen los puntos más resaltantes del texto a modo de reflexiones finales.

\section{LAS TIPOLOGÍAS EN LA INVESTIGACIÓN SOCIOLÓGICA}

\subsection{Del sentido común a las construcciones científicas}

Desde que nos hacemos "miembros de una sociedad" (Berger y Luckmann 2011: 162) aprendemos a denominar, distinguir y clasificar los objetos que componen la realidad. Parte fundamental de la socialización se basa en que las personas incorporen lo que Schütz (1995) denominaba el "acervo de conocimientos a la mano", el cual consiste en un reservorio de saberes formado en la experiencia y que otorga sentido al mundo.

Una característica de este reservorio - motivo por el cual está "a la mano"es que su movilización reviste una forma pre-reflexiva. Es decir, los individuos, en lo cotidiano, dan por sentado lo que conocen de la realidad. Si dejamos de lado estados excepcionales de reflexión, la acción social se desenvuelve en un constante pacto implícito con la realidad. Esta "actitud natural" (Schütz 1974), que suspende el razonamiento especulativo, en el nivel más profundo de la

${ }^{2}$ Parte fundamental del análisis de las formas de subjetivación corresponde al análisis de las representaciones de los actores sobre lo actuado, en este caso de las prácticas que conforman los estilos de vida. Es por ello que el diseño de la investigación incluye una parte cualitativa conformada por entrevistas abiertas a una proporción del total de la muestra cuantitativa. Creemos que mientras que la potencia de la encuesta sirve en particular para captar las observaciones de los informantes sobre el "hacer", la técnica de entrevista permite profundizar en el sentido subjetivo de su accionar, es decir en el "decir sobre el hacer" (Lahire 2006). 
conciencia, funciona como un resguardo para la persona, le brinda aquello que Anthony Giddens (2011) denomina "seguridad ontológica".

Sin embargo, existen espacios en donde esta actitud natural no constituye más que una traba, como es el caso del universo relativamente autónomo de la ciencia (Bourdieu 2000). Aquí la relación con el sentido común, constituido por aquellas anticipaciones espontáneas que Durkheim (1986) denominó "prenociones", es necesariamente antagónica. Sucede que el conocimiento científico, altamente reflexivo, solo se forma en contra de las apariencias primeras, lo cual supone como requisito para su formulación una puesta entre paréntesis de las prenociones propias y ajenas (Bourdieu et al. 2008). Este trabajo que demanda la mirada científica se resume en la célebre fórmula de Gaston Bachelard de "vigilancia epistemológica" (Bourdieu et al. 2008). Para este autor el problema del conocimiento científico debe plantearse en términos de obstáculos, que demandan del investigador un estado permanente de reflexión y cuestionamiento de sus anticipaciones espontáneas: tanto las que se crean fuera de los límites del mundo científico, como las que circulan dentro de estos ámbitos como conceptos "a la mano", listos para ser movilizados para los propósitos más diversos. Este requisito de cientificidad se redobla para el caso particular de las ciencias sociales debido a que en este campo suele reinar la imprecisión entre la opinión común y el discurso científico (Bourdieu et al. 2008).

Un elemento fundamental en la confrontación con las apariencias es el rigor conceptual. Las palabras vehiculizan presupuestos que deben ser controladas en la construcción de los objetos científicos. Es este sentido, uno de los recursos de elaboración conceptual más utilizados en la ciencia ha sido el uso de la tipología. Su origen nos remota a la teología y la anatomía comparada de la zoología, desde donde se extendió a otras áreas del conocimiento (Velasco 2014). Las tipologías constituyen recursos heurísticos que resumen en un conjunto reducido y significativo de categorías, a los individuos, grupos, instituciones, sociedades o cualquier otra unidad de análisis que es objeto de estudio (López 1996).

Las tipologías consisten en clasificaciones teórica y empíricamente fundamentadas, diseñadas y puestas a trabajar para los propósitos de la investigación. Si bien toda tipología constituye una clasificación, no toda clasificación es una tipología. Como sostiene Lozares (1990: 144)

"Elaborar una tipología y, por consiguiente, construir tipos exige una doble condición: primero la taxonomización; segundo la reducción de dicho espacio conceptual a su base mínima y la resituación de los conglomerados en esta base mínima a fin de dar entidad, aquí social, a los tipos construidos. Así, tal entidad no se reducirá a la enumeración de los valores de los atributos iniciales, sino que tales grupos ocuparán un lugar denso, sociológicamente hablando".

En cuanto su lugar en la metodología de la investigación, las tipologías se sitúan como una construcción intermedia, "entre un objeto empírico descriptivamente construido, simple generalización de la realidad tratada, 
y el objeto científico elaborado" (Lozares 1990: 140). De esta manera, la construcción de tipologías nos sitúa, primordialmente, después de la etapa de recolección de las evidencias y dentro de las tareas de organización e interpretación de la información, así como de elaboración teórica (Velasco 2014).

\subsection{Las tipologías en la investigación sociológica}

En términos generales, las tipologías suponen un trabajo de construcción conceptual que genera un orden mediante la abstracción y combinación de los elementos (las variables) que se consideren relevantes para el análisis, lo que equivale a descartar aquellas relaciones sin pertinencia para el objeto de estudio. El alejamiento de la realidad perceptiva es "el precio necesario que [el hombre de ciencia] debe pagar por el logro de la generalidad abstracta" (McKinney 1968: 20). De esta manera, la abstracción le permite al científico distinguir entre los elementos esenciales y no esenciales de un problema particular.

Sucede que la tipología como recurso científico no mide su valor en base a su grado de correspondencia con la realidad, sino por su "capacidad explicativa" (McKinney 1968: 22). En tanto construcciones, las tipologías dejan fuera información que desde otra problemática pueden resultar relevantes. Pero ello no constituye un obstáculo para la investigación, sino que es inherente a todo trabajo de construcción del objeto de estudio. Como nos recuerdan los autores de El oficio de sociólogo, "el punto de vista - dice Saussure- crea el objeto" (Bourdieu et al. 2008).

La propuesta más sistemática en lo que se refiere al uso de tipologías en las ciencias sociales es la "tipología constructiva" de McKinney (1968). Esta consiste en "una selección, abstracción, combinación y (a veces) acentuación intencionales planteadas de un conjunto de criterios que tienen referentes empíricos que sirve de base para la comparación de casos empíricos" (p.14). La tipología constructiva se sitúa en un nivel de abstracción tal que engloba las diferentes formas concretas que ha tomado este recurso en la investigación social. Así, McKinney (1968: 33) elabora una tipología de tipos según las siguientes variables: "1) relación entre el tipo y la experiencia perceptual; 2) grado de abstracción involucrado en los tipos; 3) propósito del tipo; 4) ámbito temporal del tipo; 5) ámbito espacial del tipo, y 6) función que se requiere del tipo".

Cada una de estas variables establece polaridades conceptuales, las cuales nos brindan parámetros para reconocer y clasificar las tipologías en las ciencias sociales. La primera variable establece una polaridad basada en el lugar que ocupa lo teórico y lo empírico en su derivación (ideal/extraído). La segunda distingue a los tipos según su relativa generalidad o especificidad (general/ histórico). Así, mientras más general sea el tipo, mayor serán también 
los atributos que éste deje afuera ${ }^{3}$. La tercera polaridad, fundamental en la generalización conceptual, distingue a los conceptos según se aproximen a lo regular y universal (nomotético), o lo particular (idiográfico) (científico/ histórico). La cuarta y quinta distinción se basan en la extensión temporal (atemporal/temporal) y espacial (universal/local) de los tipos. Por último, la sexta variable se centra en los fines del investigador en cuanto al grado de generalización que pretende con la tipología (generalizador/individualizador).

\subsection{Tipos ideales y tipos extraídos}

Si tomamos en consideración la primera variable, los tipos construidos se ordenan en un continuum que va desde los que se derivan principalmente desde la teoría: los "tipos ideales"; hasta los que lo hacen desde los datos empíricos: los "tipos extraídos". Es importante resaltar que ambos casos contemplan una dimensión teórica y empírica, condición sine qua non de toda formulación científica. Más bien sus diferencias se dan en el lugar que ocupa lo teórico y lo empírico en su formulación. Mientras que en el tipo ideal encontramos desviaciones de la experiencia concreta, las cuales son diseñadas precisamente para el análisis empírico por medio de su comparación con esta abstracción ideal; el tipo extraído (como lo manifiesta su nombre) se desprende de las clasificaciones que se pueden encontrar en el análisis de los datos empíricos.

Así, el "tipo ideal" de Weber (2013: 79) — sin duda el tipo más conocido y utilizado en sociología - se forma mediante el

"realce unilateral de uno o de varios puntos de vista y la reunión de una multitud de fenómenos singulares, difusos y discretos, que se presentan en mayor medida en unas partes que en otras o que aparecen de manera esporádica, fenómenos que encajan en aquellos puntos de vista, escogidos unilateralmente, en un cuadro conceptual en sí unitario".

Los tipos ideales weberianos no son otra cosa que abstracciones, dispuestas a funcionar como recursos heurísticos para la investigación. Si tomamos en cuenta la orientación neokantiana de parte de la epistemología de Weber (Gil 2014: 82), el tipo ideal no busca reflejar el "en sî", pretensión que "viola la distinción kantiana entre fenómeno y noúmeno". Más bien, constituye una construcción reflexivamente elaborada por el investigador, cuya potencia se verifica en su capacidad explicativa.

Podemos encontrar una ilustración de este recurso en la tipología de las formas de desviación que construye Howard Becker (2009) en su ya clásico libro Outsiders. Hacia una sociología de la desviación. Ésta se acerca al "tipo ideal" (en contraposición al tipo extraído) por tratarse de una clasificación conscientemente ideal y delimitada, que sirve como herramienta para el análisis.

${ }^{3}$ Como hemos sostenido, la construcción de tipos siempre excluye aspectos de la realidad y ello se cumple tanto en los más abstractos como en los más específicos. 
Al autor le interesa indagar sobre las posibles formas que puede revestir la desviación de las normas, independientemente de su actualización concreta. Para ello establece la primera dimensión sobre la que se basará su tipología: la obediencia o no de las reglas. A ésta se le agrega una más, que sirve a los propósitos hipotéticos de Becker y que complejiza las posibilidades de la tipología: la percepción de la desviación. Como podemos apreciar en la Tabla $\mathrm{N}^{\circ} 1$, la tipología bidimensional contiene cuatro casilleros en los que se encuentran todas las posibilidades de desviación en torno a esas dos dimensiones.

\section{Tabla $\mathbf{N}^{\circ}$ 1.Tipos de conducta desviada}

\begin{tabular}{|c|c|c|}
\hline & $\begin{array}{c}\text { Comportamiento } \\
\text { obediente }\end{array}$ & $\begin{array}{c}\text { Comportamiento que } \\
\text { rompe la regla }\end{array}$ \\
\hline $\begin{array}{c}\text { Percibido como } \\
\text { desviación }\end{array}$ & Falsa acusación & Desviado puro \\
\hline $\begin{array}{c}\text { No percibido como } \\
\text { desviación }\end{array}$ & Conforme & Desviado secreto \\
\hline
\end{tabular}

Fuente: elaboración propia a partir de Becker (2009).

La tipología de Becker (2009) ordena una totalidad ("la desviación de las normas"), que en una primera aproximación se le presenta al investigador como amorfa y diversa, en torno a las dimensiones (variables) que constituyen los parámetros que ordenan su objeto de estudio. A su vez, esta tipología le sirve al autor para enunciar hipótesis acerca de patrones de comportamiento similares entre quienes se ubican en cada uno de los casilleros de la tabla. Hay que notar que este recurso no constituye una reificación del fenómeno en ambas dimensiones, sino que se presta como una estrategia metodológica para explorar todas las posibilidades lógicas de la construcción conceptual, las cuales reclaman su contrastación empírica mediante el análisis de los casos concretos.

En el polo opuesto al tipo ideal se ubica el tipo extraído. Este a diferencia del primero, no se crea premeditadamente de acuerdo a principios pre establecidos (como agotar las posibilidades lógicas para Becker), sino por medio de fuentes empíricas. Es debido a su fundamentación empírica a priori, que la construcción de tipos extraídos suele estar acompañada de métodos estadísticos ${ }^{4}$. Mientras en los tipos ideales prevalece la acentuación de los rasgos más típicos, el tipo extraído se basa en el cálculo de promedios y la identificación de lo común: por un lado, las comparaciones se establecen con los extremos, y por el otro, estas se realizan con respecto a las tendencias centrales (McKinney 1968). Es por esta derivación, teórica en el tipo ideal y empírica en el tipo extraído, que el grado de

${ }^{4}$ No obstante, también existen métodos de análisis cualitativo -algunos de ellos muy complejos como el "análisis cualitativo comparativo" (Becker 2009)- que permiten elaborar tipos extraídos empíricamente fundamentados. 
ideación e interpretación cobra un peso más significativo en el primero.

Sin embargo, como ya hemos sostenido, el tipo ideal y el tipo extraído no deben tomarse como opuestos, sino más bien como polos dentro de una gama. El reconocimiento de esto último es crucial ya que, si bien el lugar de la teoría y la fundamentación empírica los diferencia, ambos involucran necesariamente un trabajo de construcción del objeto que, como elabora Marx (2007: 21) a propósito del método de la economía política, va de lo abstracto a lo concreto:

"Lo concreto es concreto porque es la síntesis de múltiples determinaciones, por lo tanto, unidad de lo diverso. Aparece en el pensamiento como proceso de síntesis, como resultado, no como punto de partida, aunque sea el verdadero punto de partida, y, en consecuencia, el punto de partida también de la intuición y de la representación. (...) las determinaciones abstractas conducen a la reproducción de lo concreto por el camino del pensamiento".

En lo que sigue desarrollaremos una vía de construcción de tipos extraídos en base a la aplicación de tos técnicas de análisis estadístico. Como hemos señalado arriba, la construcción de tipologías concierne principalmente a la etapa de la investigación en que se realizan las tareas de organización e interpretación de la información. Es por ello que comenzaremos con la exposición del marco analítico básico que guiará la interpretación de la información recabada. Nuestro propósito para lo que sigue del artículo es doble: ejemplificar al tipo extraído con un caso concreto y exponer los pasos que hemos seguido para su construcción.

\section{LA CONSTRUCCIÓN DE UN TIPO EXTRAÍDO: LOS ESTILOS DE VIDA DE HOMOSEXUALES MASCULINOS}

En esta sección expondremos los resultados preliminares de una investigación en curso sobre las formas de subjetivación de homosexuales masculinos en Lima. Por tratarse de un estudio actualmente en etapa de elaboración, los resultados que presentaremos aquí pueden variar en la presentación final de informe. Sin embargo, el anticipo de los hallazgos que presentamos aquí no cambiará de manera sustancial.

En la primera parte de este apartado brindamos una contextualización de la situación de la diversidad sexual del Perú, lo cual nos permite enmarcar los resultados que se discuten más adelante. En lo que sigue se expone la metodología de la investigación y el marco analítico que enmarca la construcción tipológica, indispensable en la construcción del objeto científico. Finalmente, cerramos esta sección con la exposición de la secuencia que seguimos para la construcción del tipo extraído en base a dos técnicas de análisis estadístico: el análisis de correspondencias múltiples (ACM) y la clasificación jerárquica ascendente $(\mathrm{CAJ})$, así como una última sección dedicada a la corroboración de hipótesis mediante esta tipología. 


\subsection{Una aproximación a la situación actual de la diversidad sexual en el Perú}

Como se manifiesta en los informes que la Red Peruana TGLB y la ONG Promsex han venido elaborando anualmente desde el 2008 sobre la situación de la población LGBTI en el Perú, las experiencias de este sector de la sociedad siguen marcada por la discriminación, el odio y la violencia. Además, esta situación de vulnerabilidad se redobla por la inacción del Estado, que se manifiesta en la falta de normativa para sancionar los actos de discriminación y violencia que sufren estas poblaciones (Red Peruana TLGB y Promsex 2016).

Si bien la iglesia católica y las iglesias evangelistas han encabezado la oposición a las iniciativas para garantizar la seguridad y extender los derechos a las poblaciones LGBTI, difícilmente se podría sostener que actúan en contra de los valores del grueso de la población. Sucede que, si bien en los últimos años se ha registrado una evolución positiva en la aceptación de las poblaciones no heterosexuales (Cruz y Guibert 2015), los niveles de intolerancia en el Perú continúan siendo muy altos. En un estudio elaborado el año 2012 por el Instituto de Opinión Pública de la Pontificia Universidad Católica del Perú sobre la percepción de la población sobre las personas trans y gay en ocho regiones del Perú, se muestra que en los últimos años existe una evolución positiva hacia la aceptabilidad de la diversidad sexual. Ello se traduce en que la mayoría de la población se muestra favor de apoyar leyes que protejan a estas personas. Sin embargo, cuando en esta misma encuesta se pregunta sobre su inclusión en instituciones como la familia y la enseñanza básica, la mayoría se muestra en contra. A su vez, es significativo que un $25.6 \%$ del total de encuestados sostienen que no les gustaría tener de vecinos a homosexuales, lo cual ubica a la homosexualidad como la cuarta categoría más estigmatizada debajo de "drogadictos", "gente con antecedentes penales" y personas que beben mucho/ dados a la bebida" (IOP-PUCP 2012).

Una de las características de la homofobia en el Perú, así como en otros contextos sociales, es su vinculación con la masculinidad. Como sostiene Óscar Guasch (2006: 21) "la masculinidad hegemónica se construye mediante el sexismo y la homofobia. Las mujeres y los homosexuales (pero también los minusválidos o los miedicas) son los otros a quienes se atribuye un estatus social interior" (p.21). Los jóvenes son instados al ejercicio de la violencia contra quienes demuestran actitudes disruptivas con la "masculinidad hegemónica"5. Ello explica la temprana aparición de la homofobia en la vida de las personas,

${ }^{5}$ Este concepto alude a un tipo ideal de masculinidad que domina por sobre las demás masculinidades y feminidades. Así “'la masculinidad hegemónica' no es un tipo de personalidad fija, siempre igual en todas partes. Se trata más bien de la masculinidad que ocupa la posición hegemónica en un modelo dado de relaciones de género, posición que siempre es discutible" (Connell, 2003). Por masculinidad, el concepto permite diversificar las maneras en que esta se presenta en la sociedad, mientras que la "hegemonía" alude al "dominio de la sociedad que ejerce un grupo determinado, no mediante el uso de la fuerza bruta sino a través de una dinámica cultural que se extiende a la vida privada y los ámbitos sociales" (Giddens, 2007). 
usualmente en la etapa escolar. Aquí la homosexualidad se dispone como una amenaza, un dispositivo en la constitución de la masculinidad que, al colocar al hombre en una posición simbólicamente femenina, constituye la materialización de lo abyecto (Fuller 2001). Ello se refleja en un estudio sobre el bullying u hostigamiento homofóbico en Chile, Guatemala y Perú, titulado Era como ir todos los días al Matadero (2013). Para el caso peruano, se muestra la prevalencia del discurso y la violencia homofóbica entre los jóvenes. Más aún, resulta significativo que las agresiones que sufren los jóvenes son toleradas por las autoridades y profesores, lo cual legitima estas formas de violencia, con implicancias más allá del ambiente escolar. Se halló que en el Perú el $44 \%$ de los encuestados $(\mathrm{n}=900)$ sostuvo haber sufrido bullynig en la escuela, pero este porcentaje crece para quienes se identifican como no heterosexuales (68\% vs 43\%); a su vez, es significativamente más frecuente la experiencia de haber sido víctimas de expresiones de bullying homofóbico entre los no heterosexuales (48\% vs 13\%) (Cáceres y Salazar 2013: 17).

En el Perú la problemática de la diversidad sexual ingresó con mucha fuerza en el debate público en los últimos años con motivo de la campaña para la aprobación del proyecto de ley que contempla la unión civil no matrimonial para personas del mismo sexo ${ }^{6}$. Con la excepción de la denominación de "matrimonio" y del derecho a la adopción, en lo esencial este proyecto otorgaba a los denominados "compañeros civiles" casi todos los derechos de un matrimonio entre heterosexuales: podrían formar una sociedad de gananciales, recibirían el mismo tratamiento y tendría los mismos derechos que un pariente de primer grado y recibirían protección contra la violencia familiar y otros beneficios de protección social que brinda el Estado. Sin embargo, tras una larga campaña en medios desde distintos sectores de la sociedad, la Comisión de Justicia y Derechos Humanos del Congreso de la República del Perú decidió, por amplia mayoría, archivar el proyecto de ley.

A pesar del resultado desfavorable, el trabajo que los distintos colectivos y sectores de la sociedad civil lograron visibilizar como nunca antes la situación de las poblaciones LGBTI en el Perú. Sin embargo, no es muy arriesgado afirmar que en la actualidad la homofobia, sus implicancias y las ideas que trae aparejadas se han logrado difundir a la gran mayoría de la ciudadanía, ya sea para negar la pertinencia del problema o para descalificar una argumentación (o a su portavoz). En este sentido, a las diversas formas de discriminación y exclusión reconocidas por la población, como el "racismo" o el "machismo" o "sexismo", se le ha incorporado la "homofobia", como lo demuestra no solo su vasta cobertura en los principales medios de comunicación masiva, sino su incorporación en la agenda de la Defensoría del Pueblo del Perú ${ }^{7}$, órgano

\footnotetext{
6 Se puede consultar el proyecto de ley en el siguiente enlace: http://www2.congreso.gob.pe/ Sicr/TraDocEstProc/Contdoc02 2011 2.nsf/d99575da99ebfbe305256f2e006d1cf0/588055827c0 8debd05257be4005f45ec/\$FILE/PL02647120913.pdf

7 Sobre el involucramiento de la Defensoría del Pueblo en la implementación de políticas que garanticen y protejan los derechos de las personas LGBTI, consultar nota
} 
constitucional autónomo del Estado peruano creado para defender los derechos fundamentales.

\subsection{La metodología del estudio}

La exposición que sigue se basa en los resultados de un cuestionario virtual auto administrado y distribuido por internet durante los últimos meses del 2014 e inicios del 2015, a una muestra de 566 personas del universo de asistentes a páginas web y/o redes virtuales de socialización erótica homosexual. Nuestra elección de internet como el espacio para distribuir el cuestionario se basó en que en la actualidad las redes y páginas web de intercambio homoerótico congregan a una gama muy amplia de homosexuales masculinos, muchos de ellos alejados de la cultura gay limeña y global (Leal 2011; Mowlabocus 2010; Luengo 2010), siendo así un espacio particularmente apto para los fines de nuestra investigación. El muestreo fue por conveniencia y se contó con la participación de ocho personas encargadas de la distribución del enlace con la encuesta.

\subsection{El marco analítico: homosexuales masculinos y estilos de vida}

La "homosexualidad" como problemática tiene una historia marcada por la medicalización del erotismo (vuelto "sexualidad"), y la secularización (en clave de patología) de los antiguos delitos contra la moral cristiana (Foucault 2008; Di Segni 2013). Como lo ha evidenciado Foucault (2008), el homosexual surge en alusión a un tipo de persona, a una "especie", más que al acto en sí. $\mathrm{Si}$ en la moral cristiana el énfasis estaba en el acto prohibido (el pecado de la sodomía), en el siglo XIX la medicina, luego la psiquiatría, la psicología y la sexología se centraron en el agente. El interés que despertó su estudio devino en la construcción de una persona con una condición patológica. No tardaron en surgir teorías acerca de los orígenes y causas de esta perversión, muchas de ellas difundidas en la cultura popular, las cuales nutrieron imágenes estereotipadas y profundamente negativas del homoerotismo.

Como otros tantos estigmas sociales, la homosexualidad se constituyó como aquello que Hughes (1946: 357) denomina "estatus maestro", que consiste en una característica que "tiende a dominar, en las situaciones más cruciales, cualquier otra característica que podría ir en contra de este" la diversidad dentro del universo de los hombres que practican el homoerotismo, los homosexuales fueron reducidos a algunas características "típicas". Como

de prensa en el siguiente enlace: http://sinetiquetas.org/2016/08/31/peru-defensoria-delpueblo-recomienda-implementar-politicas-proteger-derechos-lgbti/

${ }^{8}$ Traducción propia del original en inglés: "tends to overpower, in most crucial situations, any other characteristics which might run counter to it". 
sostiene Gagnon (1980: 27), la alusión a la homosexualidad ha traído consigo

"inferencias acerca de las clases de familias de que proceden; de las clases de vidas sexuales que llevan; de sus gustos en el vestir, en el arte, en la música y en decoración interior; de la forma en que hablan, de los tipos de trabajo que prefieren, y de sus orientaciones religiosas o con respecto al tiempo libre".

En las últimas décadas los estudios alrededor de la diversidad sexual, tanto de aquellas disciplinas ligadas a la medicina y la salud ya mencionadas, como de los programas de investigación de las ciencias sociales, han buscado precaverse de las prenociones del sentido común y del discurso médico patologizante. Estos cambios, a su vez, fueron impulsados por el activismo de los movimientos LGBTI $^{9}$ (Di Segni 2013), los cuales lucharon por que prevalezca una concepción de la sexualidad más cerca de lo cultural que de lo biológico.

Uno de los efectos de estas transformaciones en la academia ha sido una suerte de limpieza conceptual en torno a las denominaciones que se utilizan para referirse a toda la gama de colectividades que, como sostiene Butler (2007: 72), no mantienen "relaciones de coherencia y continuidad entre sexo, género, práctica sexual y deseo". Sin embargo, existen diferencias en las formas en que se incorpora la diversidad sexual en las investigaciones. Por un lado, están aquellas que, en tanto buscan incluir la enorme gama de experiencias e identidades, recurren a las denominaciones y clasificaciones propias de los sujetos que conforman la investigación ${ }^{10}$. Se trata, en términos de Harris (1996) ${ }^{11}$, de estudios con una mirada emic sobre la sexualidad: ponen el acento en los sentidos y nociones que manejan los "nativos". Destacan las investigaciones realizadas desde disciplinas como la sociología y la antropología, las cuales suelen recurrir a enfoques etnográficos en entornos específicos, tanto físicos como virtuales (Motta 2001; Perlongher 1993; Guasch 1995; Sívori 2005; Meccia 2006 2011; Leal 2011; Luengo 2009). Se caracterizan por situar en primer plano la forma en que los individuos hacen de su sexualidad una experiencia, la manera en que desarrollan identidades alrededor de esta y la conformación de subculturas sexuales, algunas de ellas muy específicas y con un alto grado de especialización en cuanto a las lógicas que las integran (Green 2014) ${ }^{12}$. A su vez, estos trabajos se sitúan en diálogo con el activismo político

9 Las siglas LGBTI refieren a lesbianas, gays, bisexuales, trans e intersexuales, y tiene como objetivo resaltar la diversidad sexual. En la actualidad existen muchas variantes, según diferentes contextos regionales. En este artículo se utiliza LGBTI debido a que es la denominación más difundida en el Perú.

10 Por ejemplo, Sívori (2005), en el libro que presenta los resultados de la etnografía que realizó en la ciudad de Rosario, utiliza las denominaciones nativas "locas", "chongos" y "gays" para referirse a quienes integran esta subcultura homosexual. Un caso similar es el de Guasch (1995), con su libro La sociedad rosa, donde el autor analiza las transformaciones ocurridas en el mundo homosexual y gay español.

11 Harris (1996) retoma la distinción que establece el lingüista Kenneth Pike entre los "resultados estructurales" obtenidos por el análisis fonético, y los no estructurales de la fonética (p. 491), para distinguir entre la perspectiva de los observadores (etic) y la de los observados (emic).

12 El nivel de especialización de los entornos sexuales es uno de los componentes más 
LGBTI y los debates teóricos sobre la sexualidad y el género. Particularmente con la denominada "teoría queer", corriente post estructuralista inspirada en los trabajos de Foucault, Derrida y Lacan, y cuyo principal referente lo constituyen los trabajos de la filósofa norteamericana Judith Butler.

Por el otro lado, están aquellos estudios que, con fines analíticos, recurren a categorías estandarizadas. Aquí resaltan los estudios epidemiológicos sobre el $\mathrm{VIH} /$ sida. Parten de una perspectiva etic, en tanto se privilegian las nociones del observador por sobre las de los observados. Si bien muchos de estos incluyen métodos cualitativos e incluso enfoques etnográficos, por lo general se trata de trabajos que privilegian una mirada objetivista sobre la sexualidad, lo cual se refleja en la denominación que alude a hombres homo/bisexuales y que ha servido de piedra de toque en sus investigaciones: "hombres que tienen sexo con hombres" (HSH) (Cáceres 2004; Fernández-Dávila et al. 2008; Klavs et al. 2009; Semaille 2009). Según Cáceres (2004: 40) este concepto

"(1) correctamente alude a que es su conducta homosexual, y no su identidad sexual u otra asignación externa de «naturaleza homosexual» esencial, la que sirve para definir al grupo desde el punto de vista de la salud pública, en principio; (2) resulta un término inclusivo para muchos tipos de 'HSH' con distintas experiencias personales; y (3) se posiciona como una denominación técnica totalmente asimilable por los programas gubernamentales de lucha contra el SIDA".

El autor también reconoce que esta denominación ha sido objeto de muchas críticas desde distintos sectores de la sociedad civil porque la consideran como una muestra de homofobia por parte de sus principales impulsores (Cáceres 2004). Quienes utilizan esta categoría sostienen que "intenta superar la supuesta homogeneidad que presentan ciertas categorías identitarias y revelar asi $\mathbb{\otimes}$ la enorme diversidad y heterogeneidad a partir de una compleja interrelación entre deseo sexual, prácticas y comportamientos sexuales, redes sociosexuales y roles de género" (Barreda et al. 2010: 3).

A pesar de sus limitaciones, creemos que el concepto $\mathrm{HSH}$ tiene como ventaja dejar abiertas las posibilidades de subjetivación o el desarrollo de identidades a partir de los deseos y prácticas sexuales, debido precisamente a su énfasis en el comportamiento. Consideramos que esto último es una vía muy potente para pensar el fenómeno de la sexualidad en términos más dinámicos, con un énfasis en los cambios, lo cual lleva a interesarse en procesos o etapas de desarrollo (Plummer 1996). Esta dimensión diacrónica es usualmente dejada de lado en los estudios etnográficos, que suelen enfocarse en identidades y subjetividades que, en cierto sentido, parecen más bien cristalizadas. En este sentido, si lo que se busca es la reconstrucción de procesos de subjetivación, el énfasis en los comportamientos permite lleva a dejar abiertas las posibilidades de

resaltantes de las culturas sexuales modernas según Green (2014). Ello se evidencia en la proliferación de espacios como los bares para solteros, clubes de S y M, salas de chat eróticas, bares de trans, etc. 
subjetivación para su indagación.

Es por ello que en esta investigación nos servimos del énfasis comportamental del HSH. Sin embargo, para fines analíticos, optamos por la denominación "homosexual", ya que consideramos que tiene la virtud de retener lo comportamental, la orientación sexual, además de encontrarse cerca de las denominaciones emic específicas a la población que comprende esta investigación. En este sentido, nuestro concepto de "homosexualidad" se asemeja al de HSH en tanto nos referimos a aquellos hombres que dirigen sus deseos y prácticas eróticas hacia personas de su mismo género.

Otro punto de coincidencia con el concepto HSH es que con "homosexual" incluimos tanto a quienes tienen deseos y prácticas dirigidas exclusivamente a personas de su propio género, como a quienes también lo hacen con el género opuesto, comúnmente consideradas como "bisexuales". Su inclusión dentro de esta categoría, comúnmente reservada para quienes tienen relaciones sexuales exclusivamente homosexuales, se debe principalmente a dos motivos. En primer lugar, la experiencia de las personas bisexuales tiene muchos puntos de contacto con la de aquellos que practican exclusivamente el homoerotismo, por lo que su delimitación nunca es clara. Por un lado, ambas formas de sexualidad conllevan la posibilidad de su ocultamiento. En palabras de Goffman (1995), homosexuales y bisexuales tienen la posibilidad de ser "desacreditables" y no directamente "desacreditados", en tanto tienen la posibilidad de disimular su estigma. Por otro lado, los bisexuales, en su búsqueda de relaciones homoeróticas, suelen compartir espacios con quienes suscriben a una identidad homosexual: discotecas, bares, redes virtuales y todos aquellos lugares que conforman lo que Adam y Green (2014) denominan "circuitos eróticos". Esta copresencia involucra un elemento de socialización y cercanía con las experiencias de las personas exclusivamente homosexuales. En segundo lugar, la bisexualidad (en tanto práctica, pero también como auto atribución subjetiva) en ocasiones se dispone como una instancia previa a la asunción de una identidad homosexual (Schilz 1997) ${ }^{13}$. De esta manera, excluirlos del análisis equivaldría a descartar a parte importante de los jóvenes en etapas tempranas de la formación de su identidad sexual ${ }^{14}$.

Sin embargo, existe una diferencia significativa en el universo que comprende la denominación HSH y el de esta investigación. Mientras que el primero incluye a todos los "hombres" (determinado por el "sexo biológico") que desarrollan prácticas homosexuales, nuestro análisis solo comprende una porción de este universo, la cual se distingue principalmente por el género

13 Esta idea debe ser considerada con precaución, dado que en muchas oportunidades se utiliza el argumento de la "fase bisexual" para negar su posibilidad.

14 Ello se tradujo en que la primera pregunta del cuestionario fue por la orientación sexual del entrevistado, con las opciones: "homosexual", "bisexual" y "heterosexual". Esta pregunta sirvió, a su vez, como un filtro para excluir a los que se consideraban "heterosexuales", por posibles errores de difusión del cuestionario virtual. Si bien reconocemos que existe la posibilidad de hombres que se consideran heterosexuales a pesar de tener encuentros sexuales con personas de su mismo género, se optó por su exclusión dado el riesgo mayor de obtener filtraciones. 
de quienes incluye. De esta manera, añadimos el término "masculino" a "homosexual" para referirnos únicamente a hombres cisgénero, es decir aquellos homosexuales "no transexuales" y "no transgéneros" para quienes existe una correspondencia entre el género asignado en su nacimiento, el de sus cuerpos y el de su identidad personal (Schilt y Westbrook 2009) ${ }^{15}$.

Un elemento a resaltar en nuestro uso del término "homosexualidad masculina" es que, si bien se refiere a una persona en particular, únicamente lo hace en referencia a la dimensión sexual de su vida ${ }^{16}$. Es decir, por "homosexual" no aludimos a nada más allá del deseo y prácticas eróticas hacia el propio género. Esto en parte debido a que nuestro análisis recae precisamente en la socialización de estos deseos. En este sentido, nuestro principal interés en esta investigación consiste en analizar cómo es que los homosexuales masculinos conforman, en torno a su orientación sexual, prácticas que configuran formas de subjetivación de la sexualidad. Estas prácticas a su vez, se organizan en torno a "estilos de vida" diferenciados (Eribon 1999; Schiltz 1997; Pollak 1998).

Si bien el concepto de "estilo de vida" suele utilizarse para referirse a patrones de consumo, en el campo de la sociología nos remonta a las investigaciones de Max Weber, particularmente su sociología de las religiones (Giddens 1991). Para este autor los estilos de vida emergen de las elecciones (cursos de acción concretos) que realizan los individuos. Este componente es el que suele resaltarse en el estudio de las regularidades en el consumo. Sin embargo, Weber estableció un segundo componente en su definición, el cual puede traducirse como "oportunidades de vida" (Lebenschanchen), que se relaciona íntimamente con las posibilidades de acción disponibles para los individuos. En este sentido, los estilos de vida son un cruce entre agencia y estructura, dispositivos y disposiciones:

"los estilos de vida se basan en elecciones (Lebensführung), pero estas elecciones dependen del potencial del individuo (Lebenschanchen) para realizarlos" (Abel 1993: 554) ${ }^{17}$.

Muy cercano a esta definición de estilo de vida es la manera en que Pierre Bourdieu (2012) pone este concepto a trabajar en el sistema conceptual de una de sus obras más importantes: La distinción: criterios y bases sociales del

15 Es importante enfatizar que este concepto sirve más para diferenciar a homosexuales masculinos y lesbianas de transexuales o trasngénero, que para determinar con cierta precisión el género de quienes incluye. Esto debido a que, como sostiene Butler (1998: 314), el género no está inscrito en la naturaleza, sino que es lo que se hace diario bajo determinadas coacciones. El género suele ser más dinámico y fluido de lo que usualmente se considera bajo el binario masculino/ femenino.

16 Con ello estamos cerca de la primera definición que ofrece el diccionario de la RAE (2014) para "homosexual": "Dicho de una persona: Inclinada sexualmente hacia individuos de su mismo sexo".

17 Traducción propia del original en inglés: "In sum, lifestyles are based on choices (Lebensfihrung), but these choices are dependent upon the individual's potential (Lebenschancen) for realizing them. Lebensfiihrung is not a lifestyle; rather, it is the element of choice in Weber's concept of lifestyles". 
gusto. Aquí la construcción del espacio de los estilos de vida de las distintas fracciones de clase en Francia se basa en los gustos y prácticas culturales: todas aquellas elecciones que se desprenden del juicio del gusto y que se basan en clasificaciones a modo de esquemas cognitivos, las cuales se traducen en formas de clasificación y jerarquización social. Bourdieu demuestra cómo las disposiciones estéticas tienen una correspondencia con la desigual distribución de recursos culturales y económicos ${ }^{18}$.

En esta misma línea, pero tomando en cuenta el lugar que ocupa la elección y la individuación en las sociedades contemporáneas, Anthony Giddens (1991) ha propuesto una actualización del concepto de estilo de vida. Según su formulación, en un universo post-tradicional la elección se desenvuelve cada vez menos como una opción: "no solo todos seguimos estilos de vida, sino que en un sentido importante estamos forzados a hacerlo, no tenemos otra elección que elegir"19 (Giddens 1991: 81).

Existe un componente en la formulación de estilos de vida propuesta por Giddens que merece especial atención, particularmente para nuestros propósitos. En la actualidad los espacios en los que se desarrolla la vida social son cada vez más diversos y segmentados, lo cual multiplica las formas de estilos de vida que se abren a los individuos. Pero éstos no suelen moverse solo en uno de estos ambientes, sino que son cada vez más quienes transitan por distintos espacios (a veces, contradictorios) en los que incorporan modos de ver, pensar y actuar. Por esta razón Giddens (1991: 83) propone hablar de "sectores de estilos de vida":

"En parte debido a que la existencia de múltiples ambientes de acción, las elecciones de estilos de vida y las actividades suelen ser segmentarias para el individuo: los modos de acción seguidos en un contexto pueden más o menos variar con los que adopten en otros. Llamaré a estos segmentos sector de estilo de vida"20.

Estos sectores corresponden a actividades en las que determinados cursos de acción se circunscriben en determinados límites espacio-temporales. Una formulación similar acerca de esta pluralidad en los repertorios de los individuos es la de Bernard Lahire (2006) en su propuesta de sociología disposicional y contextual. Como heredero de Bourdieu, particularmente de su teoría de la práctica, pero a la vez desde una perspectiva crítica con respecto a sus análisis, Lahire (2006) establece una ruptura con el sistema de disposiciones transferibles que compone el habitus de Bourdieu. Para este autor, Bourdieu no habría podido reconocer lo plural en el seno del individuo, en parte debido a que su sociología

18 En esta correspondencia se basa la "hipótesis de homología" que constituye el núcleo de su indagación (Baranger 2006).

19 Traducción propia del original en inglés: "we all not only follow lifestyles, but in an important sense are forced to do so -we have no choice but to choose".

20 Traducción propia del original en inglés: "Partly because of the existence of multiple milieux of action, lifestyle choices and activities very often tend to be segmental for the individual: modes of action followed in one context may be more or less substantially at variance with those adopted in others. I shall call these segments lifestyle sectors". 
de la práctica - junto con su concepto de habitus - no habría sufrido ninguna variación al extrapolarse desde el contexto de una sociedad tradicional, y rural como la Cabilia argelina, a las grandes urbes europeas.

Por ello Lahire (2004) propone considerar al habitus como una forma específica de disposiciones sistemáticas desarrolladas en determinados entornos, igualmente específicos: los campos. Lo que ocurre fuera de estos espacios relativamente autónomos es igualmente relevante para el análisis social, ya que aquí también se forman disposiciones cognitivas y comportamentales, dispuestas a funcionar en y mediante su activación en determinados contextos ${ }^{21}$. Si traemos esta concepción al terreno de la sexualidad, la orientación sexual, fundamental en la conformación de la "identidad sexual", no es sino una dimensión de la vida social en la que los actores incorporan determinadas disposiciones eróticas, estéticas y relacionales, dispuestas a funcionar en entornos específicos, con pares y dinámicas propias.

En este sentido, la discusión de los estilos de vida trae consigo el análisis de todas aquellas prácticas reflexivamente organizadas, entre las que encontramos la dimensión erótica/sexual, con sus componentes culturales, sociales e identitarios. Aquí cuando hablamos de estilos de vida de homosexuales masculinos nos referimos a la forma que adopta la dimensión sexual en sus vidas. Esta puede, en un extremo, quedar circunscrita a determinados contextos de acción. Aquí pensamos no solo en quienes viven su sexualidad de una forma clandestina, sino en aquellos para quienes la sexualidad constituye un componente secundario con respecto a otras identidades sociales. Pero en el otro extremo, también puede involucrar prácticas que exceden a la esfera erótica y que se plasman en apuestas estéticas, culturales y políticas. Es decir, la homosexualidad puede involucrar más que los deseos y prácticas eróticas. Si seguimos a Seidman (2002), en el primer caso el homoerotismo sería el soporte para la formación de una "identidad secundaria", mientras que en el último caso lo sería para una "identidad nuclear".

Sea como fuere, el estudio de la sexualidad, y la homosexualidad masculina en particular, requiere prestar atención a sus componentes propiamente sociales y culturales. Como sostiene Foucault (2015: 178), lo que vuelve más turbadora la homosexualidad es "el modo de vida homosexual mucho más que el acto sexual mismo".

21 En Lahire (2004) las disposiciones formadas en la experiencia, son actualizadas en determinados entornos. Así, los contextos funcionan como resortes de la acción, sin los cuales las disposiciones incorporadas quedan latentes. 


\subsection{Las dimensiones de los estilos de vida}

Como señalamos anteriormente, nuestro interés radica en determinar los diferentes estilos de vida que siguen los homosexuales masculinos en Lima. Es por ello que la primera interrogación que surge es: ¿cuáles son los indicadores que incluiremos en el análisis? Esta decisión es fundamental, ya que sobre estos indicadores y variables, cobrará forma el objeto de estudio (Lebaron y Bonnet 2014: 131). Como se verá más adelante, en la técnica de análisis estadístico que utilizaremos, análisis de correspondencias múltiples (ACM), esto se traduce en la elección de las variables principales. A continuación, presentamos las dimensiones de los estilos de vida y sus correspondientes indicadores:

Lúdicalerótica. Esta dimensión consiste en la participación en espacios, tanto físicos como virtuales que constituyen los lugares de socialización principalmente erótica y lúdica de la subcultura homosexual limeña: discotecas, bares, saunas, salas de video y páginas o redes virtuales de interacción para homosexuales.

Relacional. Aquí las variables incorporadas se componen de dos sub dimensiones: la afectiva y la de las amistades. En la primera nos interesa saber si es que han incorporado el homoerotismo dentro del terreno afectivo, por lo que el indicador se basa en el establecimiento o no de relaciones de pareja. La dimensión de las amistades es fundamental en la experiencia histórica de la homosexualidad masculina. Esta esfera ha funcionado para muchos homosexuales, no solo como un refugio ante el rechazo de familiares y amigos, sino como una especie de "familia sustitutiva" (Eribon 1999: 57).

Cultural/estética. Esta dimensión se compone de todas aquellas prácticas de consumo cultural de productos que incorporan la temática de la diversidad sexual. Aquí entra el conocimiento de cine, series para la televisión, teatro, literatura y páginas web o blogs.

También hemos incorporado en el análisis una sub dimensión que corresponde a la asistencia a centros comunitarios y organizaciones políticas gay. Esta variable puede corresponder tanto a la dimensión cultural/estética como a la relacional.

\subsection{El espacio de los estilos de vida}

El ACM es una de las técnicas de análisis multivariado más utilizada en la investigación sociológica. Su popularidad se desprende del uso que le dio Pierre Bourdieu en sus investigaciones, particularmente en La distinción (2012). El origen de esta técnica la encontramos en los trabajos del estadístico francés Jean-Paul Benzécri y la escuela francesa de análisis de datos (Baranger 2009: 97). Se trata de una generalización del análisis factorial de correspondencias, la cual es especialmente apropiada para el análisis de tablas de contingencia de grandes dimensiones, lo cual brinda una representación geométrica de las relaciones entre filas y columnas (Cuadras 2014: 161). En consecuencia, 
mientras que el análisis factorial de correspondencias realiza el análisis en una tabla bidimensional simple, el análisis del ACM lo hace sobre una tabla disyuntiva completa, compuesta por todos los cruces entre las modalidades de dos o más variables.

El objetivo del ACM es describir las relaciones entre las variables en un espacio de pocas dimensiones con una pérdida mínima de información en provecho de la interpretación de los resultados en forma geométrica en el mapa perceptual. Por ello el momento fundamental en el ACM, así como en la construcción tipológica, lo constituye la elección de las variables que se incorporarán al análisis.

\subsection{La interpretación del ACM}

El espacio de representación del ACM, el mapa perceptual, está construido por la interrelación de todas las modalidades de las variables activas. Cada eje del mapa perceptual corresponde a un factor (dimensión), que explica una parte de la variabilidad total de la nube de puntos, la cual lleva el nombre de inercia o fi cuadrado. En el ACM esta medida es fija y no nos sirve para interpretar las relaciones entre las variables (función que sí tiene en el análisis de correspondencias). Así, la inercia total es un número fijo que depende del número de variables y sus modalidades. Sin embargo, su descomposición en cada factor nos permite elegir las dimensiones que se considerarán en el análisis ${ }^{22}$.

Como se muestra en el Gráfico $\mathrm{N}^{\circ} 1$, los dos primeros factores explican el $52.28 \%$ del total de la inercia de la nube de puntos. Si observamos el descenso de la inercia explicada desde el tercer factor, podemos inferir que en estas primeras dimensiones se hallan las relaciones que nos brindarán información más relevante.

22 Como sostiene Baranger (2009), "la decisión de cuántos factores hay que tomar en el ACM no se puede resumir en un conjunto de reglas preestablecidas" (p. 108). 


\section{Gráfico $\mathbf{N}^{\circ} 1$. Histograma de los valores propios del ACM}

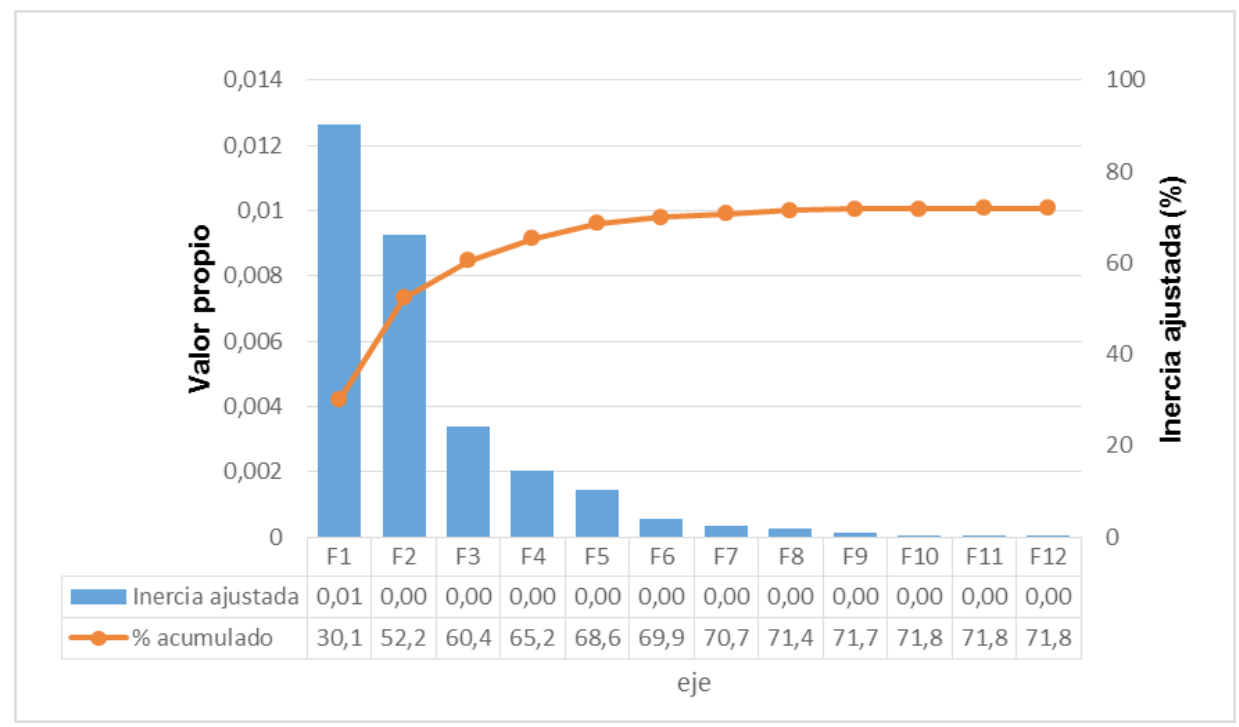

Fuente: elaboración propia según datos de encuesta (2014).

En el ACM tanto los individuos como las variables se representan en el mapa. Nos concentraremos en el mapa perceptual de las variables principales que presentamos a continuación (ver Gráfico $\mathrm{N}^{\circ} 2$ ). Si nos centramos en el primer factor, que explica el $30.18 \%$ de la inercia, las modalidades de las variables se distribuyen de tal forma que en los extremos del mapa se sitúan los indicadores que corresponden a la dimensión cultural/estética de los estilos de vida. De esta manera, vemos que conforme nos movemos a la derecha del mapa encontramos (en orden ascendente) las modalidades que representan el consumo de las distintas manifestaciones culturales del universo gay.

Una de las reglas de interpretación del ACM consiste en que la proximidad entre dos modalidades de variables distintas indica que éstas están asociadas. Tomando esto en consideración, podemos comprobar que la cercanía de los indicadores que componen la dimensión cultural/estética en sus mayores valores (BLOGS-3, BLOGS-4, LIT-1, TV-2, CINE-2) demuestra lo interrelacionadas que están estas prácticas de consumo cultural.

En cuanto al segundo factor, que explica el $22.10 \%$ de la inercia, vemos que las modalidades que se distribuyen a lo largo de este son aquellas que componen la dimensión lúdica/erótica de los estilos de vida. En el extremo superior, y conforme vamos descendiendo, encontramos los indicadores de no asistencia a discotecas (DISC-0) y bares (BAR-0), mientras que en el extremo inferior están las que denotan una participación activa en estos espacios (SAU-1, VID-1, BAR3, BAR-2, DISC-3). 
Gráfico $\mathbf{N}^{\circ}$ 2. Análisis de correspondencias múltiples. Variables principales en los dos primeros factores

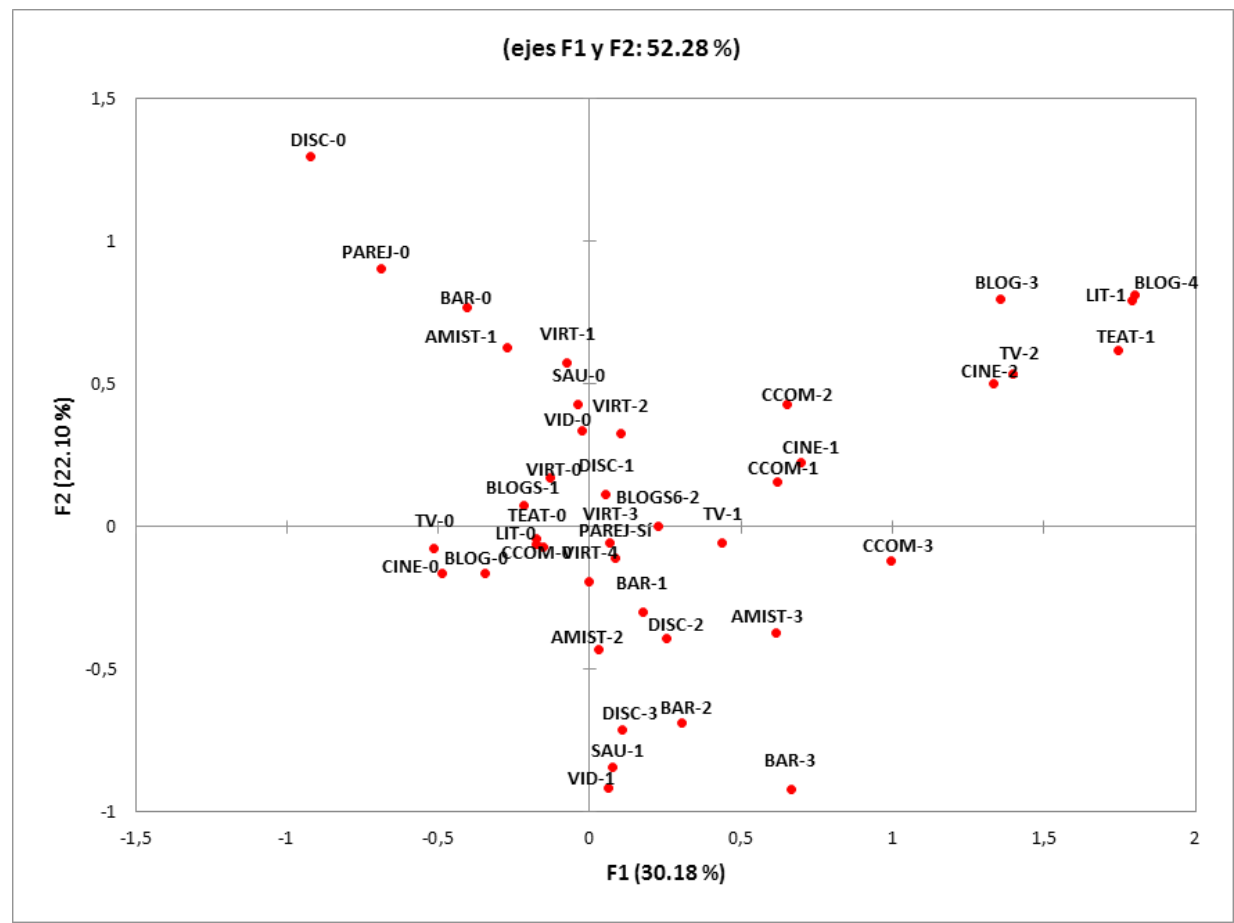

Fuente: elaboración propia según datos de encuesta (2014).

Finalmente, si nos enfocamos en las modalidades que representan a la dimensión relacional de los estilos de vida (AMIST y PAREJ), vemos que éstas se distribuyen en forma diagonal, situándose las que consisten en el no establecimiento de relaciones de pareja y de amistades relativamente próximos y situados al lado derecho superior del mapa, mientras que sus contrarios lo hacen en el lado opuesto.

\subsection{Los tipos extraídos de la homosexualidad masculina}

Si bien este breve análisis nos ha permitido conocer la forma en que se asocian estas variables, nuestro principal interés radica en la elaboración de una tipología de tipos extraídos de individuos en torno a estas asociaciones. Para realizarlo utilizaremos las coordenadas de las proyecciones de cada uno de los individuos en el mapa perceptual como valores que se incorporarán en un análisis de clusters: la clasificación ascendente jerárquica (CAJ).

Jean-Paul Benzécri (1992), el creador del ACM, considera a la CAJ como 
un "método complementario al análisis de correspondencias" 23 . Se trata de una técnica de clasificación estadística, la cual consiste en el agrupamiento de los objetos en clases de modo que sean lo más homogéneas dentro de ellas y lo más heterogéneas entre ellas. Ello lo realiza mediante un algoritmo que agrupa los elementos más cercanos de manera iterativa hasta que todos los elementos están incorporados en un solo grupo. Como resultado se construye una jerarquía de clasificaciones (ver Anexo No2), lo cual deviene en la conformación de grupos o clases, que para el caso de este estudio son tres ${ }^{24}$. Con el fin de conocer dónde se sitúan estas clases en el mapa perceptual hemos sustituido las etiquetas de la proyección de cada individuo por su clase en el mapa perceptual (ver Gráfico $\mathrm{N}^{\circ} 3$ ).

Gráfico $\mathbf{N}^{\circ}$ 3. Análisis de correspondencias múltiples. Proyección de los individuos en base a la CAJ

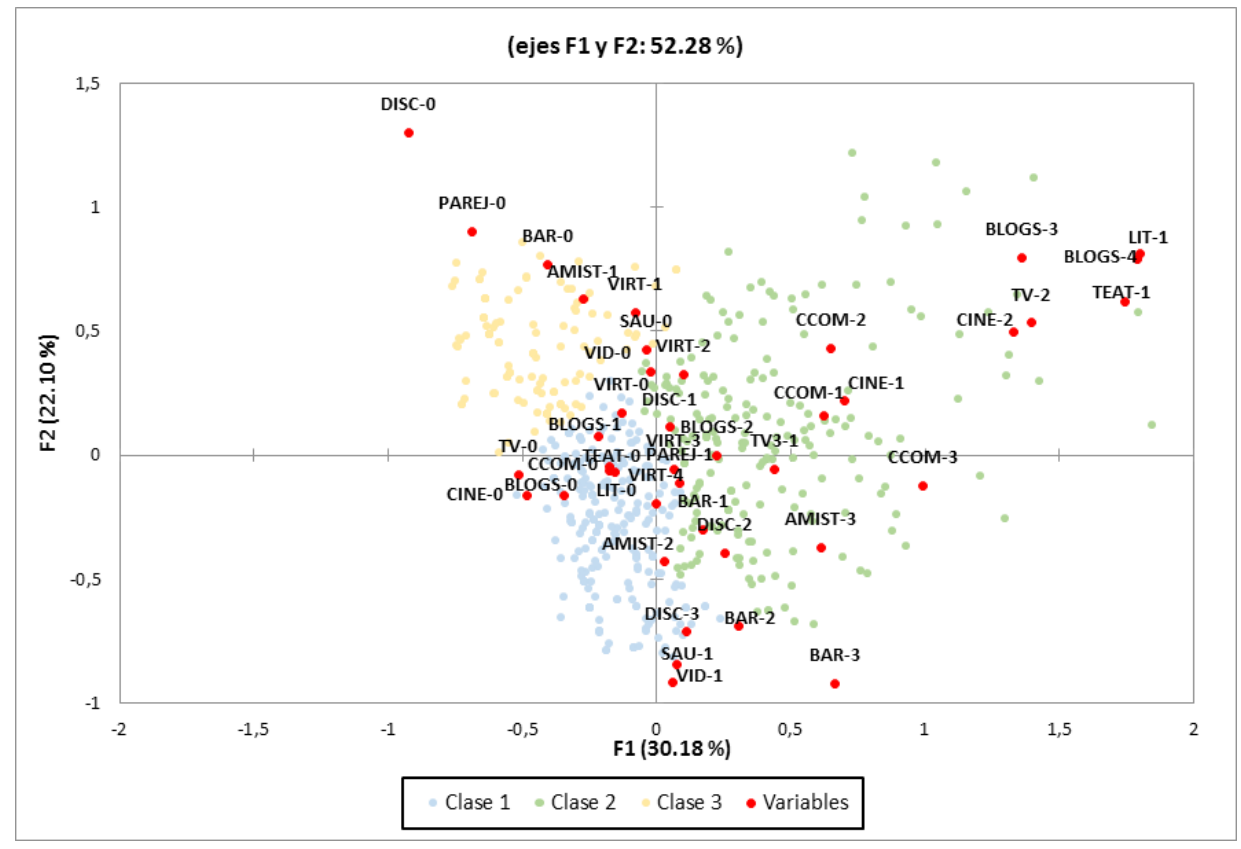

Fuente: elaboración propia según datos de encuesta (2014).

23 Traducción propia del original en inglés: "Agglomerative hierarchical clustering is a companion method to correspondence analysis".

${ }^{24}$ El número de clases depende de la estructura de los datos, pero es elegida por el investigador. En este caso hemos elegido el criterio de truncación automática, el cual establece tres clases y satisfacen la regla que sostiene que "la varianza entre las clases debe ser mayor a la varianza en cada clase" (Le Roux y Rouanet 2014:114 citado en Bergström y Dalberg 2014: 251). 
Así, los individuos que corresponden a la Clase 1 se encuentran distribuidos en la parte inferior izquierda del mapa de correspondencias, lo cual — basados en el análisis previo de los factores- podemos inferir que se trata de un grupo que se distingue de los demás por su activa participación en los circuitos lúdico/eróticos del mundo gay. En cuanto a la Clase 2 (los más dispersos en el mapa), aquí encontramos a los individuos que se caracterizan por su consumo cultural/estético de contenidos gay. También observamos que este grupo no se halla alejado de los circuitos eróticos, por lo que podemos argumentar que esta agrupación corresponde a quienes incorporan su orientación sexual en sus prácticas en los espacios más diversos: desde la socialización erótica, hasta el interés por el cine o la participación en centros comunitarios gay.

Por último, el tercer grupo, que es el que reúne a menos personas ${ }^{25}$, corresponde a individuos que se encuentran alejados de la participación en los espacios que componen la dimensión lúdico/erótica de los estilos de vida, así como del consumo que involucra su forma cultural/estética. A ello hay que sumarle que, en contraste con las demás clases, se encuentran distribuidos muy cerca a los indicadores que representan el no relacionamiento, tanto a nivel de amistades como de pareja, con pares homosexuales (la dimensión relacional).

\subsection{Las tipologías y las hipótesis}

Con el análisis precedente hemos presentado una forma de construcción tipológica post hoc denominada "extraída", en contraste con los tipos que se desprenden de la ideación, como en el ejemplo que presentamos en la primera parte del artículo sobre los tipos de desviación que propone Howard Becker. Sin embargo, una de las funciones principales de las tipologías, presente tanto en los "tipos ideales" como en los "tipos extraídos", es que sirven para la corroboración de hipótesis.

Una de las hipótesis de nuestra investigación señala que la formación de los estilos de vida de los homosexuales masculinos se relaciona con la publicidad de la orientación sexual hacia las personas que componen su entorno: familia, amigos y compañeros de trabajo. De esta manera, sostenemos que la proximidad a las dimensiones que componen los estilos de vida constituyen oportunidades - en el sentido weberiano del término- para que los actores consideren deseable la "salida del closet" 26 . Para verificar esta hipótesis hemos tomado a las clases que componen la tipología de estilos de vida como una variable, para luego efectuar pruebas de hipótesis Chi cuadrado con cada una de las variables que miden la publicidad de la orientación sexual.

25 La primera clase agrupa a 239 individuos, la segunda a 202 y la tercera a 115.

26 Seidman (2004), entre otros, sostiene que la narrativa del comming out (lo que en castellano se conoce como "salir del closet" o "salir del armario") es indispensable para comprender la formación de la homosexualidad como identidad. 
Tabla $\mathbf{N}^{\circ}$ 2. Clase y publicidad de la orientación sexual

\begin{tabular}{|c|c|c|c|}
\hline Padre & La conoce & No la conoce & Total \\
\hline 1 & 41.54 & 58.46 & 100.000 \\
\hline 2 & 48.47 & 51.53 & 100.000 \\
\hline 3 & 24.74 & 75.26 & 100.000 \\
\hline Total & 40.44 & 59.56 & 100 \\
\hline \multicolumn{4}{|l|}{ p-valor 0.001 alfa 0.05} \\
\hline Madre & La conoce & No la conoce & Total \\
\hline 1 & 59.49 & 40.51 & 100.000 \\
\hline 2 & 67.48 & 32.52 & 100.000 \\
\hline 3 & 39.18 & 60.82 & 100.000 \\
\hline Total & 58.02 & 41.98 & 100 \\
\hline \multicolumn{4}{|l|}{ p-valor $<0.0001$ alfa 0.05} \\
\hline Hermanos & La conoce & No la conoce & Total \\
\hline 1 & 62.56 & 37.44 & 100.000 \\
\hline 2 & 72.39 & 27.61 & 100.000 \\
\hline 3 & 39.18 & 60.82 & 100.000 \\
\hline Total & 61.10 & 38.90 & 100 \\
\hline \multicolumn{4}{|l|}{ p-valor $<0.0001$ alfa 0.05} \\
\hline Amigos het. & La conoce & No la conoce & Total \\
\hline 1 & 83.08 & 16.92 & 100.000 \\
\hline 2 & 90.18 & 9.82 & 100.000 \\
\hline 3 & 54.64 & 45.36 & 100.000 \\
\hline Total & 79.56 & 20.44 & 100 \\
\hline \multicolumn{4}{|l|}{$p$-valor $<0.0001$ alfa 0.05} \\
\hline Compañeros de trabajo & La conoce & No la conoce & Total \\
\hline 1 & 65.13 & 34.87 & 100.000 \\
\hline 2 & 74.23 & 25.77 & 100.000 \\
\hline 3 & 36.08 & 63.92 & 100.000 \\
\hline Total & 62.20 & 37.80 & 100 \\
\hline
\end{tabular}

Fuente: elaboración propia según datos de encuesta (2014).

Como podemos observar en las pruebas de hipótesis que aparecen en la Tabla $\mathrm{N}^{\circ}$ 2, la publicidad de la orientación sexual se relaciona significativamente con las clases que hemos construido. En las tablas se observa que quienes componen la Clase 2, constituida por quienes desarrollan estilos de vida que se distinguen por la dimensión cultural/estética, tienden a comunicar a sus familiares, amigos e incluso a sus compañeros de trabajo sobre su orientación 
sexual en una proporción significativamente mayor que las demás clases, particularmente con respecto a los que están incluidos en la Clase 3. Esta última, compuesta por quienes no construyen estilos de vida en torno a su sexualidad, comprende a quienes tienden a no comunicar este aspecto de sus vidas ${ }^{27}$.

De la evidencia presentada podemos corroborar que la formación de estilos de vida en torno a la orientación sexual condiciona la comunicación de la orientación sexual. Es decir, las disposiciones cognitivas y comportamentales adquiridas en la socialización con pares homosexuales, y mediante el consumo de ciertos productos culturales, se traducen en un interés por salir del closet.

\section{REFLEXIONES FINALES}

En la primera parte de este artículo presentamos las principales características y posibilidades que brinda el recurso de la tipología para la investigación en ciencias sociales. Para ello nos hemos basado, principalmente, en la propuesta de "tipo construido" de McKinney y su tipología de tipologías en torno a las dimensiones que ordenan las posibilidades para este recurso analítico. De estas, nos hemos detenido particularmente en la polaridad compuesta por el "tipo ideal" weberiano y el "tipo extraído", debido a su relevancia para la tipología que presentamos.

En la segunda parte del artículo, nuestro propósito ha sido presentar una vía de construcción de tipos extraídos por medio de la elaboración teórico-conceptual del objeto y, fundamentalmente, del análisis de la información recabada del instrumento cuantitativo por medio dos técnicas de análisis multivariado: el ACM y la CAJ. Con la aplicación del ACM, hemos evidenciado su potencialidad para la construcción del objeto de estudio mediante la incorporación de las variables principales que estructuran el mapa perceptual.

En cuando a la CAJ, su uso nos permitió efectuar una clasificación de los individuos incluidos en el análisis, en base a sus coordenadas en el espacio construido por el ACM. La aplicación de esta técnica de clasificación (cluster) constituye el punto de llegada de la construcción de los tipos extraídos.

Sin embargo, el análisis no termina aquí, ya que más que una forma de construir/ organizar la información, las tipologías científicas cobran sentido en tanto sirven a los fines de la investigación, ya sea en la lógica del descubrimiento (v. gr. en el hallazgo de relaciones no consideradas), o en lo que respecta al contraste de hipótesis. De esta manera, como toda teoría, método o técnica de investigación, la construcción de tipologías no es más ni menos que una herramienta para el avance del conocimiento científico.

27 Ello se manifiesta, v. gr., en que la Clase 3 no comunica a amistades heterosexuales sobre su orientación sexual en más del doble de la media (45.3\%/20.44\%). 


\section{BIBLIOGRAFÍA}

ABEL, T. y COCKERMAN, W. C. (1993): "Lifestyle or Lebensführung? Critical Remarks on the Mistranslation of Weber's "Class, Status, Party", The Sociological Quarterly, 34(3), pp. 551-556.

ADAM, B. D. y GREEN, A. I. (2014): "Circuits and the social organization of sexual fields", en Sexual Fields: toward a sociology of collective sexual life, Chicago, Chicago University Press, pp. 123-142.

BARANGER, D. (2009): Construcción y análisis de datos. Introducción al uso de técnicas cuantitativas en la investigación social, Buenos Aires, Posadas.

BARREDA, V., CARBALLO-DIEGUEZ, A., MARONE, R., BALÁN, I., PANDO, M. Á., \& AVILA, M. M. (2010): "Prevención Del Vih/sida en los circuitos de levante HSH: una asignatura pendiente", Sexualidad, Salud Y Sociedad: Revista Latinoamericana, (6), pp. 41-62.

BECKER, H. (2009): Outsiders. Hacia una sociología de la desviación, Buenos Aires, Siglo XXI Editores.

BENZÉCRI, J. (1992): Correspondence analysis handbook, New York, Marcel Dekker.

BERGER, P.L. y LUCKMANN, T. (2011): La construcción social de la realidad, Buenos Aires, Amorrortu editores.

BERGSTRÖM, Y. \& DALBERG, T. (2014): "Education, social class and politics: the political space of Swedish youth in Uppsala", en Bourdieu and Data analysis. Methodological principles and practice, Bern, Peter Lang AG, pp. 227-252.

BOURDIEU, P. (2012): La distinción. Criterio y bases sociales del gusto, Madrid, Editorial Taurus.

BOURDIEU, P. (2003): El oficio de científico. Ciencia de la ciencia y reflexividad. Curso del College de France 2000-2001, Barcelona, Editorial Anagrama.

BOURDIEU, P., PASSERON, J. \& CHAMBOREDON, J. (2008): El oficio de sociólogo: presupuestos epistemológicos, México, Editorial Siglo XXI.

BUTLER, J. (2007): El género en disputa. El feminismo y la subversión de la identidad, Barcelona, Paidós.

BUTLER, J. (1998): “Actos performativos y constitución del género: un ensayo sobre fenomenología y teoría feminista", Debate Feminista, vol.18.

CÁCERES, C. F. (2004): "La Epidemiología, el SIDA y la Sexualidad: Las Persistentes Brechas entre la policía Sanitaria y la promoción de la ciudadanía en salud sexual", en Ciudadanía Sexual en América Latina: Abriendo el Debate, Lima, Universidad Peruana Cayetano Heredia, pp. 35-45.

CÁCERES, C. F. y SALAZAR, X. (ed.) (2013): "Era como ir todos los días al matadero...": El bullying homofóbico en instituciones públicas de Chile, Guatemala y Perú. Documento de trabajo, Lima, IESSDEH, UPCH, PNUD, UNESCO.

CRUZ, M. y GUIBERT, Y. (2015): Actitudes hacia la homosexualidad en el Perú, Lima: IOP PUCP (Cuadernos de Investigación, $\mathrm{N}^{\circ} 11$ ).

COHEN, N. y GÓMEZ, G. (2011): "Las tipologías y sus aportes a las teorías y la producción de datos", Revista Latinoamericana de Metodología de la Investigación Social. No1. Año 1. Abril - Sept. de 201, pp. 36-46.

CONNELL, R. W. (2003): Masculinidades, México, Universidad Nacional Autónoma de México.

DI SEGNI, S. (2013): Sexualidades. Tensiones entre la psiquiatría y los colectivos militantes, Buenos Aires, Fondo de Cultura Económica. 
DURKHEIM, E. (1986): Las reglas del método sociológico, México, Fondo de Cultura Económica.

ERIBON, D. (1999): Reflexiones sobre la cuestión gay, Barcelona, Editorial Anagrama. FERNÁNDEZ-DÁVILA, F., SALAZAR, X., CÁCERES, C., MAIORANA, A., KEGELES, COATES, T., MARTINEZ, J. (2008): "Compensated sex and sexual risk: sexual, social and economic interactions between homosexually-and heterosexually-identified men of low income in two cities of Peru", Sexualities 11 (3), pp. 352-374.

FOUCAULT, M. (2008): La historia de la sexualidad 1. La voluntad de saber, Buenos Aires, Siglo XXI.

FOUCAULT, M. (2015): La ética del pensamiento: para una crítica de lo que somos, Madrid, Biblioteca Nueva.

FULLER, N. (2001): Masculinidades. Cambios y permanencias. Varones de Cuzco, Iquitos y Lima, Lima, Fondo editorial de la PUCP.

GAGNON, J. H. (1980): Sexualidad y conducta social. México, Editorial Pax.

GIDDENS, A. (2011): La constitución de la sociedad. Bases para la teoría de la estructuración, Buenos Aires, Amorrortu editores.

GIDDENS, A. (2000): La transformación de la intimidad. Sexualidad, amor y erotismo en las sociedades modernas, Madrid, Ediciones Cátedra.

GIDDENS, A. (1991): Modernity and self-identity. Self and society in the late modern age, California, Stanford University Press.

GIDDENS, A. (2007): Sociología, Madrid, Alianza Editorial.

GIL, F. (2014): "Introducción", en Economía y Sociedad, México, Fondo de Cultura Económica, pp. 9-109.

GOFFMAN, E. (1995): Estigma. La identidad deteriorada, Buenos Aires, Amorrortu.

GREEN, A. I. (2014): "Introduction. Toward a sociology of collective sexual life", en Sexual fields: toward a sociology of collective sexual life, Chicago, The University of Chicago Press, pp. 1-24.

GUASCH, Ó. (1995): La sociedad rosa, Barcelona, Anagrama.

GUASCH, Ó. (2006): Héroes, científicos, heterosexuales y gays. Los varones en perspectiva de género, Barcelona, Bellaterra.

HARRIS, M. (1996): El desarrollo de la teoría antropológica. Historia de las teorías de la cultura, México, Siglo XXI.

INSTITUTO DE OPINIÓN PÚBLICA DE LA PUCP (2012): Percepción de la población general hacia la población trans y gay/HSH, en las regiones de Lima, Callao, Ica, La Libertad, Lambayeque, Loreto, Ucayali y San Martín, Lima, IOPPUCP.

KLAVS I., BERGANT N., KASTELIC Z., LAMUT A., KUSTEC T. (2009): "Disproportionate and increasing burden of HIV infection among men who have sex with men in Slovenia: surveillance data for 1999-2008”, Euro Surveill, 14(47):pii=19419.

LAHIRE, B. (2006): El espíritu sociológico, Buenos Aires, Manantial.

LAHIRE, B. (2004): El hombre plural: los resortes de la acción, Barcelona, Bellaterra.

LEAL, S. (2011): La pampa y el chat. Aphrodisia, imagen e identidad entre hombres de Buenos aires que se buscan y encuentran mediante internet, Buenos Aires, Antropofagia. 
LEBARON, F. \& BONNET, P. (2014): "Classification, social classes and cultural practices: a GDA approach through the Bourdieu's sociology of culture", en Bourdieu and data analysis. Methodological principles and practice, Bern, Peter Lang AG, pp. 127-164.

LÓPEZ, P. (1996): "La construcción de tipologias: metodología de análisis", Papers: Revista de Sociologia, (48), 9-29.

LOZARES, C. (1990): "La tipología en sociología, más allá de la simple taxonomía: conceptualización y cálculo”, Papers, 34(1990), pp. 139-163.

LUENGO, F. (2009): Masculinidades no dominantes: Una Etnografía de Gaydar, disponible en página web [consulta: 2-11-2016].

MARX, K. (2007): Elementos fundamentales para la crítica de la economía política. Borrador 1857-1858, volumen I, México, Siglo XXI Editores.

MECCIA, E. (2006): La cuestión gay. Un enfoque sociológico, Buenos Aires, Gran Aldea Editores.

MECCIA, E. (2011): Los últimos homosexuales. Sociología de la homosexualidad y la gaycidad, Buenos Aires, Gran Aldea.

MCKINNEY. J. (1968): Tipología constructiva y teoría social. Buenos Aires, Amorrortu Editores.

MOTTA, A. (2001): "Entre lo tradicional y lo moderno: La construcción de identidades homosexuales en Lima", en De amores y luchas. Diversidad sexual, derechos humanos y ciudadanía, Lima, Centro de la Mujer Peruana Flora Tristán, pp. 143164.

MOWLABOCUS, S. (2010): Gaydar culture: Gay men, technology and embodiment in the digital age, Londres, Ashgate Publishing Company.

PERLONGHER, N. (1991): La prostitución masculina, Buenos Aires, La Urraca.

POLLAK, M. (1987): “La homosexualidad masculina o: ¿la felicidad en el ghetto?”, en Sexualidades occidentales, Buenos Aires, Paidós.

PLUMMER, K. (1996): "Symbolic interactionism and the forms of homosexuality", en Queer Theory/Sociology, Cambridge, Mass, Blackwell, pp. 75-98.

RED PERUANA TLGB y PROMSEX (2016): Informe anual sobre derechos humanos de personas trans, lesbianas, gays y bisexuales en el perú 2015-2016, disponible en http://promsex.org/images/docs/Publicaciones/InformeTLGB2015al2016.pdf [consulta 3-11-2016]

REAL ACADEMIA ESPAÑOLA. (2014): Diccionario de la lengua española, 23. ${ }^{a}$ ed., Madrid, Espasa.

SEMAILlE, C., CAZEIN, F., LOT F., PILlONEL, J., LE VU, S., LE STRAT, Y., BOUSQUET, V., VELTER A, BARIN, F. (2009): "Recently acquired HIV infection in men who have sex with men (MSM) in France, 2003-2008", Euro Surveill, 14(48): pii=19425.

SCHILT, K. y WESTBROOK, L. (2009): "Doing gender, doing heteronormativity. 'Gender normals', transgender people, and the social maintenance of heterosexuality", Gender \& Society, vol. 23 No.4, pp.440-464.

SCHILTZ, M. A. (1997): "Young homosexual itineraries in the context of HIV: Establishing lifestyles, Population, an English selection, 10区 année, n², pp. 417445.

SCHUTZ, A. (1995): El problema de la realidad social. Escritos I. Buenos Aires, Amorrortu Editores.

SEIDMAN, S. (2004): Beyond the closet. Transformation of gay and lesbian life. London, Psychology Press. 
SÍVORI, H. (2005): Locas, chongos y gays: sociabilidad homosexual masculina durante la década de 1990, Buenos Aires, Antropofagia.

VELASCO, M. (2014): "Un acercamiento al método tipológico en sociología”, en Observar, escuchar y comprender. Sobre la tradición cualitativa en la investigación social, México, Porrúa, El Colegio de México, FLACSO.

WEBER, M. (2013): Ensayos sobre metodología sociológica, Buenos aires, Amorrortu Editores. 
EMPIRIA. Revista de Metodología de Ciencias Sociales. N. ${ }^{\circ}$ 36, enero- abril, 2017. ISSN: 1139-5737, 